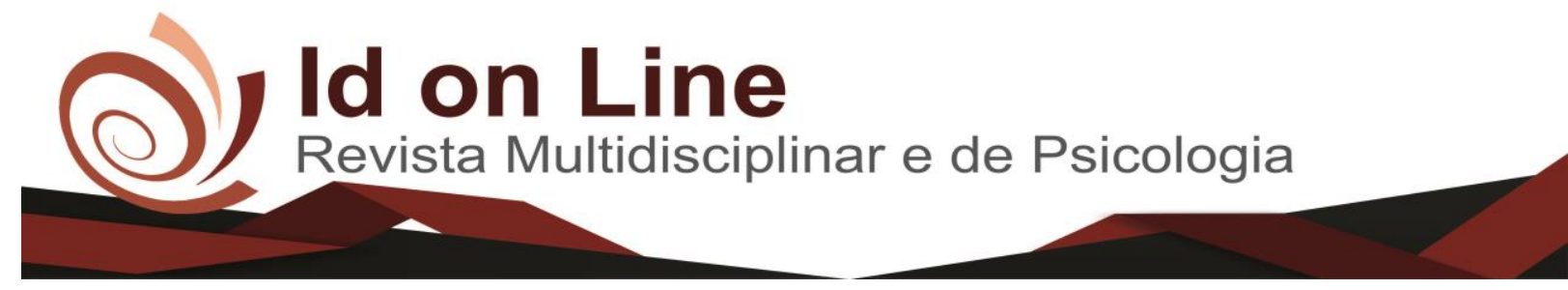

Artigo

\title{
Prevalência Relacionada à Dor Lombar em Funcionários de uma Empresa Privada
}

\author{
Virgilio Santana Júnior' ${ }^{\text {; Eloar Barros Gigante }}{ }^{2}$
}

\begin{abstract}
Resumo: A dor lombar é a segunda maior causa de visita de pacientes aos médicos, atingindo mais de $80 \%$ da população mundial e representando prejuízos financeiros para as empresas, para o governo e para os que sofrem com o problema. A maioria da população tem ou já teve alguma dor na região lombar, sendo considerada uma das principais causas de afastamentos de trabalhadores no Brasil. O objetivo pro presente estudo foi identificar a prevalência de dor lombar não específica e os fatores de riscos associados às mesmas. A pesquisa trata-se de um estudo do tipo descritivo, exploratório, com delineamento transversal e abordagem quantitativa. A pesquisa foi realizada em uma empresa privada na cidade de Vitória da Conquista - BA, no setor do Call Center e Atendimento. A amostra foi composta por funcionários com idade maior ou igual a 18 anos, de ambos o sexo, que apresentou em algum momento dor lombar em seu local de trabalho. Para coleta de dados foi utilizado um questionário sociodemográfico, o questionário de dor de MacGill e a Escala Visual Analógica - EVA. Os dados foram tabulados em forma de gráficos e tabelas e serão analisados de forma descritiva simples a partir da média e desvio padrão. A tabulação será realizada no programa Microsoft Excel 2010. A pesquisa demonstrou que a grande predominância, foi o gênero feminino contendo praticamente $73,3 \%$ da amostra. A grande maioria com $71 \%$ da amostra queixou-se de dor na região da lombar no período da pesquisa. 98\% relataram que a dor lombar não e o motivo principal para se afastar do trabalho. A respeito da avaliação do padrão da dor através do questionário MacGll, $1(\mathrm{n}=22)$ sendo pulsante a alternativa mais assinalada com $68 \% ; 2$. $(\mathrm{n}=11)$ pontada com $73 \% ; 3(\mathrm{n}=10)$ agulhada com $60 \% ; 4(\mathrm{n}=11)$ fina com $100 \% ; 5(\mathrm{n}=15)$ aperto com $87 \% ; 6(\mathrm{n}=26)$ fisgada com $100 \% ; 7(\mathrm{n}=16)$ queimação com 100\%; $8(n=7)$ formigamento com 57\%; $9(n=18)$; dolorida com $72 \%$; $10(n=6)$ Esticada com $100 \%$; no que diz respeito no caráter Afetivo os descritores mais escolhidos foram: $11(n=0) ; 12(n=0) ; 13(n=3)$ castigante com 3\%; $14(\mathrm{n}=3)$ maldita com 100\%; $15(\mathrm{n}=0)$; Na parte de descritores avaliativos o que mais teve significância foi $16(n=28)$ chata com $71 \%$; e nos descritores miscelânea foi $17(n=3)$ espalhada com $67 \%$; 18 $(n=9)$ aperta com 78\%;19 $(n=0) ; 20(n=0)$. Desta maneira, no presente estudo foi possível analisar que na empresa, pela qual foi à base da pesquisa, demonstra que há uma predominância no sexo feminino e que a grande maioria relatou sentir algum tipo de dor nos dias da pesquisa no mês de julho. Muitos relatam que a dor lombar não e o motivo principal para se afastar do trabalho e que na maioria das vezes não procuram um profissional capacitado para diagnosticar e nem tratar tal situação quando sentem.
\end{abstract}

Palavras chave: Lombar, Unimed e Funcionário.

\section{Prevalence Related to Low Back Pain in Employees of a Private Company}

\begin{abstract}
Lumbar pain is the second largest cause of patient visits to physicians, reaching more than $80 \%$ of the world's population and representing financial losses to companies, government and those suffering from the problem. The majority of the population has or already had some pain in the lumbar region, being considered one of the main causes of workers' withdrawal in Brazil. The objective of the present study was to identify the prevalence of non-specific low back pain and the associated risk factors. The research This is a descriptive, exploratory, crosssectional and quantitative approach. The research was conducted in a private company in the city of Vitória da Conquista - BA, in the Call Center and Attendance sector. The sample consisted of employees
\end{abstract}

${ }^{1}$ Fisioterapeuta. Especialista em Terapia Manual e Postural pelo CESUMAR (Centro Universitário de Maringá). Docente titular nas instituições de ensino superior: Faculdade Independente do Nordeste (FAINOR) e Faculdade de Ciência e Tecnologia (FTC) Unidade de Vitória da Conquista/BA.

${ }^{2}$ Graduação em Fisioterapia pela Faculdade Independente do Nordeste, FAINOR, Brasil. Contato: eloar.gigante@ gmail.com. 
aged 18 and over, of both sexes, who presented at some point low back pain in their workplace. A sociodemographic questionnaire was used to collect data, the Mac Gill pain questionnaire and the Visual Analogue Scale - EVA. The data were tabulated in the form of graphs and tables and will be analyzed in a simple descriptive way from the mean and standard deviation. The tabulation will be performed in the program Microsoft Excel 2010. The research showed that the great predominance was the female gender containing almost $73.3 \%$ of the sample. The vast majority of patients with $71 \%$ of the sample complained of pain in the lumbar region during the study period. $98 \%$ reported that low back pain is not the main reason for getting away from work. Regarding the evaluation of the pain pattern through the Mcgiil questionnaire, $1(\mathrm{n}=22)$ being pulsed the most indicated alternative with $68 \% ; 2 .(n=11)$ Spike with $73 \% ; 3(n=10)$ Needled at $60 \% ; 4(n=11)$ Fine at $100 \% ; 5(n=15)$ Tightening with 87\%; $6(\mathrm{n}=26)$ Spike with $100 \% ; 7(\mathrm{n}=16)$. Burning with $100 \% ; 8(\mathrm{n}=7)$ Tinnitus with $57 \%$; $9(\mathrm{n}=18)$; Painful with $72 \%$; $10(\mathrm{n}=6)$ Stretched with $100 \%$; with respect to the Affective character the most chosen descriptors were: $11(\mathrm{n}=0) ; 12(\mathrm{n}=0) ; 13(\mathrm{n}=3)$ Punishing with 3\%; $14(\mathrm{n}=3)$ Cursed with $100 \% ; 15$ $(\mathrm{n}=0)$; In the part of Descriptive descriptors, what was most significant was $16(\mathrm{n}=28)$ Chata with $71 \%$; and in miscellaneous descriptors was $17(n=3)$ Spread with 67\%; $18(n=9)$ Tightening with 78\%; $19(n=0) ; 20(n=$ 0 ). Thus, in the present study it was possible to analyze that in the company, by which it was based on the research, it shows that there is a predominance in the female sex and that the great majority reported to feel some type of pain in the days of research in the month of July. Many report that low back pain is not the main reason to move away from work and that most of the time they do not seek a qualified professional to diagnose or treat such a situation when they feel it.

Keywords: Lumbar, Unimed and Employee.

\section{Introdução}

Segundo o Instituto Nacional de Previdência Social - INSS- (2016), a dor lombar é a segunda maior causa de visita de pacientes aos médicos, só perde para a dor de cabeça. O problema atinge mais de $80 \%$ da população mundial, de acordo com dados da Organização Mundial da Saúde - OMS, e representa prejuízos financeiros para as empresas (é a maior causa de afastamento do trabalho em pessoas com menos de 45 anos), para o governo (em 2015, mais de 116 mil pessoas receberam auxílio-doença por esse motivo) e para os que sofrem com o problema (cansaço, desânimo e até mesmo estágio depressivo).

Atualmente, a grande maioria da população tem ou já teve alguma dor na região lombar, independentemente da idade. Segundo Tomé et al. (2014), a dor lombar ou lombalgia é considerada uma das principais causas de afastamentos de trabalhadores com carteira assinada no Brasil.

A dor nas costas é um agravo à saúde que acompanha o homem desde o início dos tempos, sua prevalência é elevada e ela ocorre indistintamente na população. Estima-se que de $70 \%$ a $85 \%$ da população terá algum episódio de dor nas costas no decorrer da vida (FERREIRA et al., 2014). 
Neste sentido, vários estudos epidemiológicos têm descrito o tema dor lombares. Para Teixeira et al. (2013), a dor lombar constitui a principal causa de absenteísmo ao trabalho; ultrapassa o câncer, o acidente vascular encefálico, e a síndrome da imunodeficiência adquirida como causa de incapacidade nos indivíduos da faixa etária produtiva.

Para uma empresa, por exemplo, a redução no número de homens/horas trabalhadas, devido à ausência do trabalho por períodos de tempo consideráveis, o que provoca uma perda na produtividade e na qualidade do serviço. Como solução, a empresa poderá realizar novas contratações ou pagar horas-extras aos demais funcionários, o que também irá aumentar o custo da produção. No caso do Estado, as despesas recaem sobre o Instituto Nacional do Seguro Social (INSS), e decorrem do pagamento de benefícios previdenciários (ALMEIDA, 2014).

Neste sentido, para o fisioterapeuta é de suma importância esses estudos, pois, por meio deles, os profissionais se tornam mais capacitados para traçar um tratamento mais eficaz, levando em conta a individualidade de cada paciente.

Vários são os fatores de risco para dor lombar, dentre eles, idade, nível educacional, tabagismo, obesidade, fatores psicossociais (estresse, ansiedade, depressão), classe social e fatores relacionados ao trabalho (insatisfação, tarefas monótonas, estresse, manuseio de cargas em flexão, com rotação de tronco e vibração) (FRASSON, 2016).

Neste sentido, segundo o autor torna-se importante a prevalência que geram a lombalgia por se tratar de uma patologia de alta incidência na população em geral.

Dessa forma, informações sobre a prevalência da dor lombar na população brasileira é um importante passo no sentido de revelar a abrangência e a magnitude de seus efeitos, proporcionando um direcionamento para as estratégias preventivas e de intervenção (HOY et al., 2010).

Assim a presente pesquisa teve como objetivo geral identificar a prevalência de dor lombar nos funcionários de uma empresa privada de Vitória da Conquista, Bahia. Com objetivo específicos de caracterizar o perfil sócio demográfico dos funcionários, verificar a prevalência de dor lombar nos funcionários, verificar quais os tipos de dor mais acometidos nos funcionários. 


\section{Metodologia}

Trata-se de um estudo descritivo, exploratório, transversal, caracterizado como natureza quantitativa. Foi realizado numa empresa privada, que atua na área da saúde, localizada na cidade de Vitoria da Conquista - BA.

A mostra envolveu funcionários de uma empresa privada no setor do Call Center e Atendimento. À partir de uma análise não probabilística por conveniência, a amostra foi selecionada através dos critérios de inclusão e exclusão a seguir:

- Critérios de inclusão: Funcionários com idade maior ou igual a 18 anos, de ambos os gêneros e com apresentação de algum sintoma de dor lombar em algum momento durante seu trabalho

-Critérios de Exclusão: Não serem funcionários dessa empresa; Não ter dor lombar e não ter disponibilidade de participar da pesquisa.

\section{Resultados}

No decorrer do desenvolvimento do trabalho, foram identificados 45 indivíduos para a composição da amostra. Este momento é destinado à exibição dos dados coletados e tabulados, apresentados em tabela e gráfico, oriundos dos questionários sociodemográfico, indicadores da saúde, questionário de Mac Gill e uma escala de dor (EVA).

Ao analisar a presente pesquisa é possível observar que a grande predominância no setor pelo qual foi pesquisado, foi o gênero feminino contendo praticamente $73,3 \%$ da amostra pesquisada como mostra na Tabela 1.

Outro quesito relevante no presente estudo tem relação com o peso e altura. Evidenciando $60 \%$ da amostra com peso entre 50 a $70 \mathrm{~kg}$. E 75,56\% com estatura entre 1,50 a $1,70 \mathrm{~cm}$ como mostra na Tabela 1 . Ao realizar a pesquisa observamos que os indivíduos que foram pesquisados executam suas atividades laborais sentados em seus setores específicos. 
Tabela 1. Características sócio demográficas. Vitória da Conquista/BA, 2017.

\begin{tabular}{|c|c|c|c|}
\hline Variável & Categoria & $\mathrm{N}=45$ & $\%$ \\
\hline \multirow[t]{2}{*}{ Sexo } & Masculino & 12 & $26,7 \%$ \\
\hline & Feminino & 33 & $73,3 \%$ \\
\hline \multirow[t]{4}{*}{ Idade } & 18 a 25 anos & 17 & $37,80 \%$ \\
\hline & 26 a 40 anos & 27 & $60 \%$ \\
\hline & 41 a 50 anos & 1 & $2,20 \%$ \\
\hline & 51 a 60 anos & 0 & \\
\hline \multirow[t]{5}{*}{ Peso } & $<50 \mathrm{~kg}$ & 1 & $2,20 \%$ \\
\hline & 50 a $70 \mathrm{~kg}$ & 28 & $62,20 \%$ \\
\hline & 70 a $90 \mathrm{~kg}$ & 10 & $22,20 \%$ \\
\hline & 90 a $110 \mathrm{~kg}$ & 6 & $13,30 \%$ \\
\hline & $>110$ kg & 0 & \\
\hline \multirow[t]{4}{*}{ Estatura } & $<1,50 \mathrm{~cm}$ & 0 & \\
\hline & 1,50 a $1,70 \mathrm{~cm}$ & 34 & $75,56 \%$ \\
\hline & 1,70 a $1,90 \mathrm{~cm}$ & 10 & $22,22 \%$ \\
\hline & $>1,90 \mathrm{~cm}$ & 1 & $2,22 \%$ \\
\hline Total & & 45 & $100 \%$ \\
\hline
\end{tabular}

Fonte: Dados da pesquisa. $\mathrm{N}=$ Número da amostra; $\%$ = Percentual referente ao total de prontuários consultados $(100 \%=45)$.

De acordo a presente pesquisa na Tabela 2, a maioria dos indivíduos da amostra se classifica com um tipo de saúde "nem boa, nem ruim" sendo 37,78\%.

A grande maioria explica esse resultando não tão satisfatório por conta do excesso de sedentarismo e falta da pratica de exercício físicos sendo $64,44 \%$ da amostra. Dentro dos indivíduos que realizam atividade física (35,56\% da amostra) $25 \%$ relatam praticarem algum tipo de exercício físico durante três e cinco dias da semana como mostra na Tabela 2. 
Tabela 2. Indicadores da saúde. Vitória da Conquista/BA, 2017.

\begin{tabular}{|c|c|c|c|}
\hline Variável & Categoria & $\mathrm{N}=45$ & $\%$ \\
\hline & Boa & 16 & $35,56 \%$ \\
\hline \multirow[t]{2}{*}{ Classificação da Saúde } & Muito Boa & 12 & $26,67 \%$ \\
\hline & $\begin{array}{c}\text { Nem Boa, Nem Ruim } \\
\text { Muito Ruim }\end{array}$ & 17 & $37,78 \%$ \\
\hline \multirow[t]{2}{*}{ Pratica de Exercício Físico } & SIM & 16 & $35,56 \%$ \\
\hline & NÃO & 29 & $64,44 \%$ \\
\hline \multirow[t]{7}{*}{ Se sim, Quantos dias da semana? } & 1 dia & 1 & $6,25 \%$ \\
\hline & 2 dias & 2 & $12,50 \%$ \\
\hline & 3 dias & 4 & $25 \%$ \\
\hline & 4 dias & 1 & $6,25 \%$ \\
\hline & 5 dias & 4 & $25 \%$ \\
\hline & 6 dias & 3 & $18,75 \%$ \\
\hline & 7 dias & 1 & $6,25 \%$ \\
\hline
\end{tabular}

Fonte: Dados da pesquisa. $\mathrm{N}=$ Número da amostra; $\%=$ Percentual referente ao total de prontuários consultados $(100 \%=45)$.

Ao realizar a pesquisa com funcionários de uma empresa privada de Vitória da Conquista - BA é possível observar que houve uma grande quantidade que relataram sentir algum tipo de dor na região da lombar nos últimos 15 dias do mês de julho. De acordo o a Tabela 3 e possível analisar que a grande maioria com $71 \%$ da amostra queixou-se de dor na região da lombar.

Ao verificar a Tabela 3, e possível analisar que 76\% da amostra informa que não sente

dor lombar ao iniciar algum tipo de atividade física. Esse resultado pode ser explicado, pois a grande maioria não realiza atividade física como mostra na Tabela 2.

A grande maioria com $98 \%$ relatam que a dor lombar não e o motivo principal para se afastar do trabalho. Muitos relatam que na maioria das vezes não procuram um profissional capacitado para diagnosticar tal situação, e preferem se automedicar na maioria das vezes. 
Segundo a pesquisa demonstra que $84 \%$ dos pesquisados não ver a necessidade de ir ao médico por conta das dores na região lombar. A maioria atesta que $89 \%$ da amostra não fez ou não faz nenhum tipo de tratamento para as dores lombar e demonstra que a maioria com $89 \%$ da amostra não tem problemas com a dor ao realizar suas atividades do dia a dia.

De acordo a presente pesquisa observamos que por mais que a maioria relatou sentir dor lombar nos 15 dias recorrentes da pesquisa, poucos foram os sujeitos que cogitaram se afastar do seu trabalho, mostrando que a dor lombar não era incapacitante.

Tabela 3. Indicadores de Dor Lombar. Vitória da Conquista/BA, 2017.

\section{Apresentou dor lombar nos últimos 15 dias}

Sim

Não

\section{Sente dor lombar ao iniciar algum exercicio fisico?}

$\begin{array}{lrr}\text { Sim } & 11 & 24,44 \% \\ \text { Não } & 34 & 75,56 \% \\ \text { 3 Atualmente se afasta do trabalho por conta da dor lombar? } & & \\ \text { Sim } & 1 & 2,22 \% \\ \text { Não } & 44 & 97,78 \%\end{array}$

4 Necessidade de ir ao medico por conta da dor lombar?

Sim

Não

\section{Fez ou faz algum tipo de tratamento para dor lombar?}

Sim

5

Não

6 A dor lombar atrapalha suas atividades do dia a dia?

$\begin{array}{lrr}\text { Sim } & 5 & 11,11 \% \\ \text { Não } & 40 & 88,89 \%\end{array}$

\section{Já ficou afastado do trabalho por conta da dor lombar?}

Sim

Total

Fonte: Dados da pesquisa. $\mathrm{N}=$ Número da amostra; $\%=$ Percentual referente ao total de prontuários consultados $(100 \%=45)$. 
Ao analisar o Gráfico 1 do atual estudo, percebemos que foi utilizado um mensurador da dor através da escala visual analógica conhecida popularmente com EVA, nela consta que ao perguntar o grau da dor para o sujeito da pesquisa compreendemos que a maioria respondeu como um tipo de dor grau 2 com $21,9 \%$ da amostra.

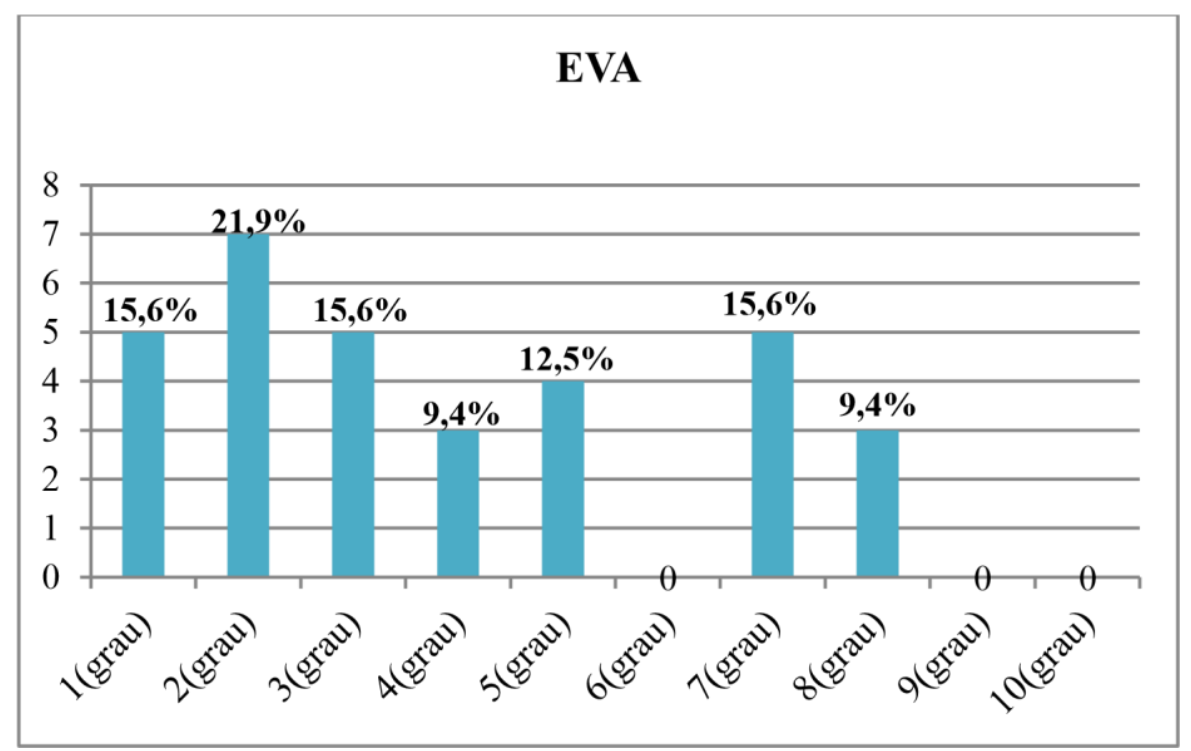

Gráfico 1. EVA (Escala Visual Analógica). Vitória da Conquista/BA, 2017.

Conforme o dado apresentado na Tabela 4 sobre a avaliação do padrão da dor através do questionário Mcgiil percebe se que os dados mais relevantes no que se diz a respeito aos descritores da avaliação sensitiva em cada subgrupo foram: $1 \quad(n=22)$ sendo pulsante a alternativa mais assinalada com 68\%; 2. $(\mathrm{n}=11)$ Pontada com 73\%; $3(\mathrm{n}=10)$ Agulhada com 60\%; $4(\mathrm{n}=11)$ Fina com 100\%; $5(\mathrm{n}=15)$ Aperto com 87\%; $6(\mathrm{n}=26)$ Fisgada com 100\%; 7 ( $n=16)$ Queimação com 100\%; $8(n=7)$ Formigamento com 57\%; $9(n=18)$; Dolorida com 72\%; $10(n=6)$ Esticada com 100\%; no que diz respeito no caráter Afetivo os descritores mais escolhidos foram: $11(n=0) ; 12(n=0) ; 13(n=3)$ Castigante com 3\%; $14 \quad(n=3)$ Maldita com $100 \% ; 15(n=0)$; Na parte de descritores Avaliativos o que mais teve significância foi $16(n=28)$ Chata com 71\%; e nos descritores miscelânea foi $17(n=3)$ Espalhada com 67\%; 18 (n=9) Aperta com 78\%;19 (n=0); $20(\mathrm{n}=0)$. 
Tabela 4. Questionário de MacGill-Avaliação do Padrão da Dor.Vitória da Conquista/BA, 2017.

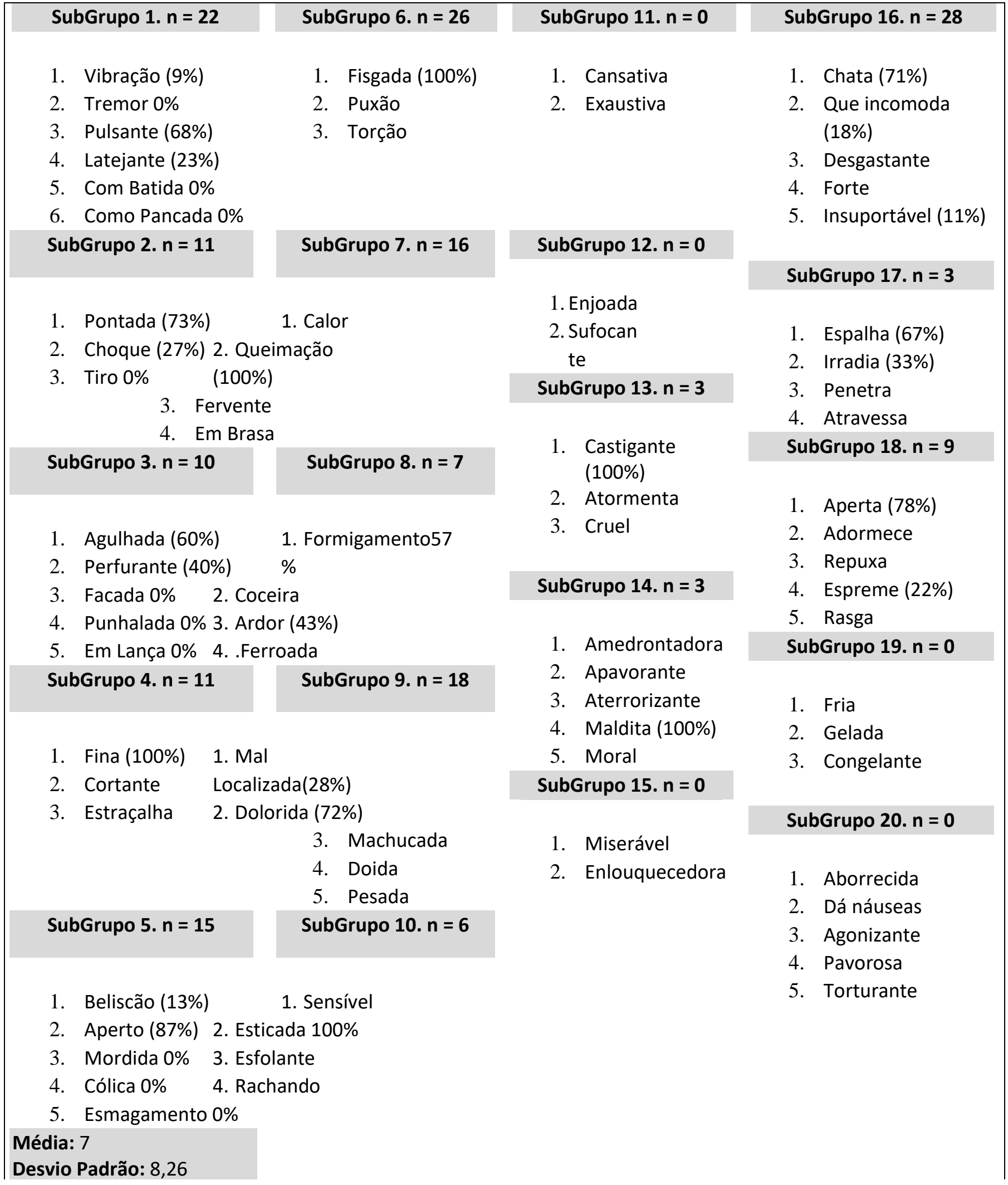

Fonte: Dados da pesquisa. N = Número da amostra). Vitória da Conquista BA, 2017. 


\section{Discussão}

As lombalgias respondem pela segunda maior causa de dor no mundo, perdendo somente para as dores de cabeça, segundo a OMS.

A dor lombar é uma condição que pode atingir até $65 \%$ das pessoas anualmente e até $84 \%$ das pessoas em algum momento da vida. Uma vez que a dor lombar apresenta caráter multifatorial que podem ser relacionada a um conjunto de causas, como, por exemplo, fatores sociodemográficos (idade, sexo, renda e escolaridade), estado de saúde, estilo de vida ou comportamento (tabagismo, alimentação e sedentarismo) e ocupação (trabalho físico pesado, movimentos repetitivos) (NASCIMENTO; COSTA, 2015).

Em Barros et al. (2011), mostra claramente que a a maioria da pesquisa é do gênero feminino e idade superior a 40 anos de idade da sua amostra. Diferente da pesquisa presente pois a idade media e entre 26 e 40 anos com $60 \%$ da amostra. Esses resultados corroboram com os achados de Lima et al. (1999), que também confirma essa prevalência maior do sexo feminino comparada com o sexo masculino na DTM, demonstrando haver diferença significativa entre os sexos, atribuindo a uma maior percepção feminina ao estímulo doloroso.

Apontam que a sobrecarga mecânica ocasionada pela dupla jornada de trabalho a qual as mulheres são submetidas parece estabelecer uma associação entre sexo feminino e a sintomatologia dolorosa. E afirmam que o excesso de gordura localizada no abdômen das pessoas com sobrepeso é apontado como causador para o desenvolvimento de dores na lombar por deslocar o centro de gravidade corporal.

Sacoo et al. (2009), diz que a postura sentada mantida por tempo excessivo pode causar carência de flexibilidade na musculatura e de redução na mobilidade articular, além de fadiga da musculatura extensora, espinhais que, aliados, comprometem a estabilidade e o alinhamento da coluna vertebral. Tais distúrbios biomecânicos são considerados importantes fatores etiológicos para o desenvolvimento de Lombalgia.

O uso excessivo da cadeira pode ocasionar um encurtamento gradual dos músculos iliopsoas e isquiostibiais, reduzindo a mobilidade articular do quadril e direcionando para frente o segmento lombar da coluna vertebral (REIS; MORO; CONTIGO, 2003). Além disso, essa posição pode causar fadiga e sobrecarga nos elementos articulares (cápsulas, ligamentos e discos intervertebrais), podendo ocasionar dessensibilização dos mecanoceptores teciduais e 
uma redução ou eliminação da força estabilizadora profunda, gerada principalmente pelo multifídos da lombar e transverso abdominal ( MACIEL; FERNANDES; MEDEIROS, 2006).

No mesmo estudo, diz que a prática de atividade física causa adaptações circulatórias e metabólicas benéficas para musculatura esquelética e tecidos, contribuindo para melhoria da postura estática e dinâmica e diminuição do risco de micro e macro lesões e incapacidades osteomusculares.

No estudo de Vitta et al. (2011), indica que a prevalência de dor lombar nos escolares foi de $19,5 \%$, sendo $64,4 \%$ nas meninas e $35,6 \%$ nos meninos, com diferença estatisticamente significante entre os gêneros. A associação entre dor lombar e as atividades sedentárias mostrou significância estatística para os fatores de risco.

Atualmente, a prática do exercício físico vem sendo compreendida no sentido de estabelecer um saber científico sobre a saúde coletiva. Um importante fator contribuinte na ausência de saúde e morte precoce é o sedentarismo que infelizmente esta tomando conta do nosso cotidiano. A ONU (Organização Mundial da Saúde) e a Federação Internacional do Esporte estimam que metade da população mundial não realize nenhum tipo de atividade física. No Brasil, cerca de $60 \%$ dos brasileiros não praticam nenhum tipo de atividade física (TOSCANO; EGYPTO, 2001).

Em um estudo longitudinal publicado por Thorbjornsson et al. (2010), foram estudados fatores ocupacionais relacionados com a lombalgia. Ao analisar os resultados demonstrou que, em ambos os sexos, tanto o sedentarismo como o trabalho com grandes cargas representam um fator de risco para a lombalgia.

Os estudos de Scopel (2013), divergem do presente estudo, pois relata que dos seus 111 pesquisados $32,4 \%$ relatam sentir dor lombar, $22,5 \%$ realizaram algum tipo de tratamento para essa dor local. A mesma atrapalha algum tipo de atividade física ao praticar com quase metade sendo este com $44.1 \%$.

Em uma pesquisa sobre a intervenção ergonômica, também discorda do presente estudo, realizado com 76 profissionais do sexo masculino de uma indústria química, identificou que os segmentos mais atingidos nos últimos três meses por sintomas musculoesqueléticos foram: a região lombar, com 19,7\%; a região torácica, com 17\%; e a região cervical, com 13\% (MIGUEZ, 2005).

Já no estudo de Picoloto e Silveira (2008), corroboram com o estudo, em pesquisa com 268 trabalhadores em uma indústria metalúrgica de Canoas-RS, com predominância do sexo masculino, observou que a região da coluna vertebral é sempre o local mais afetado com 
sintomas musculoesqueléticos, principalmente a coluna na região lombar (45\% nos últimos doze meses e $29,1 \%$ nos últimos sete dias), seguida pelos ombros $(35,1 \%$ e $21,6 \%)$, região da cervical e pescoço $(34,5 \%$ e $18,0 \%)$ e região torácica $(28,3 \%$ e $17,5 \%)$.

Segundo uma revista cientifica publicada na edição "The Spine Journal" Diz que a prática de exercícios físicos é a intervenção mais eficaz para prevenir dor na região lombar ou evitar novos episódios.

Em um estudo de Araujo (2009), aponta que apenas 16\% dos moradores de São Paulo praticam algum tipo de atividades físicas formalmente, em quantidade acima da recomendada pela OMS (Organização Mundial da Saúde), que é de pelo menos 30 minutos diários, durante cinco dias semanais. Eles são chamados de "muito ativos". O resultado é fruto de uma pesquisa realizada em 54 cidades do Estado em 2008. Foram ouvidas 2.600 pessoas com mais de 14 anos. O trabalho teve parceria com o Celafiscs, que esta a frente do programa Agita São Paulo.

Ainda neste estudo, foi confirmado que dos entrevistados, $16 \%$ são irregularmente ativos, 3,4\% afirmaram ser totalmente sedentários e 64,6\% realizam algum tipo de exercício físico informalmente.

De acordo OMS, qualquer individuo que realize algum tipo de movimento corporal realizado de forma voluntária e que gere um gasto energético acima dos níveis de repouso estará executando um tipo de atividade física, o que inclui, entre outras coisas, utilizar escadas em vez de elevadores, utilizar bicicleta ou então deixar o carro estacionado em dois ou mais quarteirões, distante do local do trabalho.

No estudo de Junior et al. (2010), diz que as dores lombares provocadas pelo ambiente de trabalho apresenta etiologia multifatorial, elevada prevalência e incidência. Caracterizada por quadro de dor com diferenças de duração e intensidade, a lombalgia pode levar à incapacidade laborativa ate mesmo chegando à invalidez. A lombalgia acarreta sofrimento aos trabalhadores, custos às empresas, aos sistemas previdenciários e assistenciais da saúde.

Segundo a revista da associação médica brasileira, a automedicação é uma prática bastante comum no Brasil, contudo se ver em outros países. A ida à farmácia representa a primeira opção procurada por muitos para tentar "resolver" um problema de saúde, e a maior parte dos medicamentos comprados é vendida sem receita médica e pode ser encontradas facilmente em farmácias, drogarias ou ate mesmo em supermercados.

A presente pesquisa vai contra os dados apresentados por Picoloto e Silveira (2008), que relata que no item de afastamento de trabalho, a região lombar também obteve a maior incidência $(22,9 \%)$, seguida da dorsal $(12,9 \%)$ e cervical $(9,6 \%)$. 
O autor evidencia a importância da região lombar, por ser uma região de sustentação do corpo e rotação do tronco, que na maioria das vezes é utilizada de forma inadequada pela má postura e carregamento de excesso de peso.

Em outra empresa, no setor de fundição e moldagem, os trabalhadores pesquisados referem à dor na região lombar como um dos maiores motivos de afastamento do trabalho com $57,89 \%$ da sua amostra (PONTES, 2005).

O tratamento multidisciplinar confirma que a eficácia para o prognóstico do paciente com dor lombar. Contudo, o médico deve ter certo cuidado ao mencionar a influência dos fatores psicossociais ao paciente, dado o entendimento leigo limitado quanto à verdadeira origem de sua dor lombar, de modo que o confronto direto com o paciente não é sábio e deve ser evitado pelo médico, pois pode resultar no abandono da terapia pois o paciente desacredita no resultado ou até mesmo gerar comportamentos inadequados e prejudiciais. A consulta deve ser feita sem desvalorizar os sintomas relatados pelo paciente (MACEDO e BLANK, 2006).

Segundo Lizier et al. ( 2012), são diversos as formas de tratamentos para a dor lombar sendo este como forma de: medicamentos (antidepressivos, anti-inflamatórios, relaxantes musculares, corticosteroides, paracetamol, dipirona, tramadol, opioides, , anticonvulsivantes), fisioterapia manipulativa e na parte de eletroterapia como: ondas curtas, ultrassom, estimulação elétrica transcutânea, laser), acupuntura e tratamento com o medico realizando o procedimento de infiltração. Entretanto, a efetividade das intervenções terapêuticas não está totalmente comprovada.

Em um estudo literário de Ferreira et al. (2010), os autores obtiveram 37 estudos que atendiam os critérios de inclusão. Desses, nove eram estudos de exercícios, sendo que oito mostraram alguma indício do efeito do exercício no tratamento de dores de lombar. Contudo o tamanho da amostra era pequeno. Somente em um estudo cujo tamanho da amostra era considerável houve evidência de que exercícios gerais melhoram a dor lombar que acabam provocando afastamento no trabalho, caso não tratadas. Os exercícios diminuem a intensidade da dor lombar e ajudam na recuperação do paciente.

No estudo de Scopel (2013), ratificam o presente estudo, na pesquisa relata que com apenas $44,1 \%$ da amostra de 111 pesquisado afirmam que sentem dores, fazendo com que prejudique em algum momento do seu dia.

No estudo de Helfenstein et al. (2010), a dor lombar é responsável por uma grande parte das faltas no trabalho, sendo estimada por estudos entre os 5 a 10 diagnósticos mais frequentes, permanecendo estável ao longo dos anos. 
A presente pesquisa vai contra os dados apresentados por Picoloto e Silveira (2008), que relata que no item de afastamento de trabalho, a região lombar também obteve a maior incidência $(22,9 \%)$, seguida da dorsal $(12,9 \%)$ e cervical $(9,6 \%)$.

De Acordo Pereira et al. (2011), há várias formas para mensurar a dor, um deles é a Escala Visual Analógica da dor (EVA). A escala consisti por uma barra horizontal em forma de régua, com uma escala de 0 a 10, onde o individuo deve informar o grau da sua dor, na qual 0 é sem dor e 10 a dor mais intensa/máxima sentida pelo individuo.

Essa escala e totalmente fácil e de rápida compreensão pelo paciente, sendo uma forma adequada para identificar a intensidade da dor presente. Sendo, porém, um instrumento unidimensional, analisa apenas a intensidade da dor, desconsiderando quaisquer outros aspectos dessa dor (MARTINEZ et al., 2011).

Dor é uma experiência subjetiva e multidimensional. O Aparecimento de instrumentos que possibilitem uma avaliação da dor nos seus diversos aspectos, é de total importância para a compreensão do quadro álgico, implementação da terapêutica e apreciação de sua eficácia. O questionário McGill é considerado um dos melhores instrumentos para a avaliação das dimensões sensitiva-discriminativa, afetiva motivacional e cognitiva-avaliativa da dor.

O questionário de dor McGill (MPQ) foi elaborado por Melzack em 1975 na Universidade McGill, Montreal, Canadá, constitui um instrumento multidimensional que visa avaliar a dor em termos qualitativos, incluindo uma anamnese do paciente. Desde então, tem sido muito utilizado em varias pesquisas sobre a dor. Esse questionário considera a dor como um fenômeno de características sensoriais, afetivas e cognitivas. Avaliar comportamento temporal e a localização, fatores de alívio e agravamento e intensidade da dor, indicam uma grande lista de descritores verbais, que caracteriza a experiência de dor (SILVA et al.,2011).

Ao analisar o estudo de Xavier et al. (2006), foi possível observar que a pesquisa objetivou comparar o comportamento quantitativo e qualitativo da dor em pacientes submetidos à toracotomia póstero-lateral (tpl). A amostra foi constituída por 18 pessoas, sendo 10 no sexo masculino e 8 no sexo feminino com idade media de 44 anos. Um dos instrumento escolhidos para analise utilizou-se o questionário McGill. Os descritores do questionário para dor McGill, escolhidos com maior freqüência pelos pacientes, foram: no componente sensorial, latejante, pontada, choque, fina e puxão; no componente afetivo, cansativa, enjoada, castigante e miserável e no componente avaliativo foi chata.

As características da dor no grupo sensorial foram mais evidentes no grupo masculino. Não foram observadas diferenças estatísticas significantes entre as respostas quantitativas da 
dor de homens e mulheres. No que diz respeito ao aspecto qualitativo, observou-se predominância dos mesmos descritores verbais do componente afetivo da dor para ambos os sexos.

No estudo de Mendes e Avelino (2016), corroboram com a presente pesquisa, eles aplicaram o questionário McGill em pacientes oncológicos os descritores mais referidos representam cada um seu subgrupo: para os descritores sensoriais $69,2 \%$ dos indivíduos falaram que sua dor é em pontada, nos descritores afetivos, $65,4 \%$ dos indivíduos disseram que sua dor é enjoada, nos descritores avaliativos $48,1 \%$ referiu a dor do câncer como insuportável e descritor miscelânea mais abordado com $40,4 \%$ foi esparrama.

\section{Conclusão}

Desta maneira, no presente estudo foi possível analisar que na empresa, pela qual foi à base da pesquisa, demonstra que há uma predominância no sexo feminino e que a grande maioria relatou sentir algum tipo de dor nos dias de pesquisa no mês de julho. Muitos relatam que a dor lombar não é o motivo principal para se afastar do trabalho e que na maioria das vezes não procuram um profissional capacitado para diagnosticar e nem tratar tal situação quando sentem.

Foi utilizada a Escala Visual Analógica - EVA, para mensurar a dor, apesar de ser um instrumento subjetivo observou que o nível de dor foi um grau reduzido.

Ao utilizar o questionário MacGill observamos que é um instrumento que identifica características sensoriais, afetivas e cognitivas. Além de avaliar comportamento temporal e a localização, fatores de alívio e agravamento e intensidade da dor, indicam uma variedade de descritores verbais, que caracteriza a experiência de dor fazendo que fique mais fácil para o paciente explicar o que de fato sente.

Visto assim, sugerem se novas pesquisas para comprovação e esclarecimento deste tema com uma amostra maior e um tempo de pesquisa mais estendido. 


\section{Referêcias}

ADORNO M. L. G. R, Avaliação da qualidade de vida com o instrumento SF-36 em lombalgia crônica. Acta Ortop Bras. 2013.

ALMEIDA, T. R. S. H. et al. (In: Memo) Hérnia de disco lombar: Riscos e prevenção, 2014.

AMARAL, João J. F. Como fazer uma pesquisa bibliográfica. Ceará: UFC, 2007. p 1

ANJOS, G. C. M.; PASSOS, V.; DANTAS, A. R. Fisioterapia aplicada à fase gestacional: uma revisão da literatura. Trabalho de Conclusão de Curso (Graduação em Fisioterapia) Universidade Federal de Pernambuco, Pernambuco, 2015.

BASTOS, A. V. B., RODRIGUES, A. C. A., MOSCON, D. C. B., SILVA, E. E. C., \& PINHO, A. P. M. Comprometimento no trabalho: Fundamentos para a gestão de pessoas. In L. O. Borges, \& L. Mourão (Orgs.), O trabalho e as organizações: Atuações a partir da psicologia (pp. 279-310). Porto Alegre: Artmed. 2013.

BRASIL, INSS, LER/ DORT Programa de Prevenção. São Paulo - SP, 2016.

BRITO, Maria Inês Meira Santos. (In:memo) Sustentabilidade e Meio Ambiente. 2014.

FERREIRA, M. S, NAVEGA, M. T. Efeitos de um programa de orientação para adultos com lombalgia. Acta Ortop Bras. 2014.

FERREIRA P, FERREIRA M, MAHER $\mathrm{C}$ et al. - Changes in recruitment of transversus abdominis correlate with disability in people with chronic low back pain. Br J Sports Med, 2010:605-614.

FRASSON, Viviane Bortoluzzi . Dor lombar: como tratar? ISBN: 978-85-7967-108-1 Vol. 1, No 9 Brasília, junho de 2016.

HOY D, March L, BROOKS P, WOOLF A, Blyth F, Vos T, et al. Measuring the global burden of low back pain. Best Pract Res Clin Rheumatol 2010;

KUIJPERS T, VAN MIDDELKOOP M, RUBINSTEIN SM ET AL. - A systematic review on the effectiveness of pharmacological interventions for chronic non-specific low-back pain. Eur Spine J, 2011

LEMOS, L. C.; MARQUEZE, E. C.; MORENO, C. R. C. Prevalência de dores musculoesqueléticas em motoristas de caminhão e fatores associados. Rev.

Bras. Saúde Ocup. v. 39, n. 129, p. 26-34. 2014.

LÜDKE, M. \& ANDRÉ, M. E. D. A. Pesquisa em Educação: Abordagens Qualitativas. São Paulo: EPU, p.45, 1986. 
NASCIMENTO PRC, COSTA LO Prevalência da dor lombar no Brasil: uma revisão sistemática Cad. Saúde Pública, Rio de Janeiro, 31(6):1141-1155, jun, 2015.

MACEDO E, BLANK VLG. Processo de trabalho e prevalência de dor lombar em motoristas de caminhões transportadores de madeira no sul do Brasil. Cad Saúde Coletiva. 2006;14:43550 .

MACIEL ACC, FERNANDES MB, MEDEIROS LS. Prevalência e fatores associados à sintomatologia dolorosa entre profissionais da indústria têxtil. Rev Bras Epidemiol 2006

MARTINEZ, J. E.; GRASSI, D. C.; MARQUES, L. G. Análise da aplicabilidade de três instrumentos de avaliação de dor em distintas unidades de atendimento: ambulatório, enfermaria e urgência. Rev. Bras. Reumatol. v. 51, n. 4, p. 299- 308. 2011.

MELZACK, R. The short-form of McGill pain questionnaire, Pain, v.30 , p. 191-7, 1987.

MINAYO, M. C. de S. Pesquisa Social: Teoria, Método e Criatividade. 6. ed. Petrópolis: Editora Vozes. 2007.

TEIXEIRA, M. J.; FIGUEIRÓ, B. J. A. Dor - Epidemiologia, Fisiopatologia, Avaliação,Síndromes Dolorosas e Tratamento. São Paulo: Grupo editorial Moreira Júnior, 2013.

TEIXEIRA, Daniella Stefany Soares. Análise da mobilidade da cintura pélvica na redução da lombalgia em pacientes com hérnia de disco. FACULDADE INDEPENDENTE DO NORDESTE - FAINOR. Monografia do Curso de Fisoterapia. Vitória da Conquista, BA, 2016.

TOMÉ F, FERREIRA C. B, CORNELLI, R. J. B., CARVALHO, A. R. Lombalgia crônica: comparação entre duas intervenções na força inspiratória e capacidade funcional. Fisioter Mov. 2012

REIS PF, MORO ARP, CONTIJO LA. A importância da manutenção de bons níveis de flexibilidade nos trabalhadores que executam suas atividades laborais sentados. Rev Prod On Line 2003.

SACCO ICN, ALIBERTI S, QUEIROZ BWC, PRIPAS D, KIELING I, KIMURA AA et al. A influência da ocupação profissional na flexibilidade global e nas amplitudes angulares dos membros inferiores e da lombar. Rev Bras Cineantropom Desempenho Humano 2009.

THORBJORNSSON CB, ALFREDSSON L, FREDRIKSSON K, MICHELSEN H, PUNNET L, VINGARD E, et al. Physical and psychosocial factors related to low back pain during a 24year period. A nested case-control analysis. Spine 2000.

TOSCANO, J.J.O and EGYPTO, E. P.A influência do sedentarismo na prevalência de lombalgia. Rev Bras Med Esporte[online]. 2001.

VAN MIDDELKOOP M, RUBINSTEIN SM, VERHAGEN AP Et al. - Exercise therapy for chronic nonspecific low-back pain. Best Practice Res Clin Rheumatol, 2010. 
XAVIER, T.T; TORRES, G.V and ROCHA, V. M.Qualitative and quantitative aspects of pain in lateral posterior thoracotomy patients. Rev. LatinEnfermagem [online]. 2006.

\section{Como citar este artigo (Formato ABNT):}

SANTANA JÚNIOR, Virgílio; GIGANTE, Eloar B. Prevalência relacionada à dor lombar em funcionários de uma empresa privada. Id on Line Revista Multidisciplinar e de Psicologia, 2017, vol.11, n.38, p. 879-896. ISSN: 1981-1179.

Recebido: 11.11.2017

Aceito: 13.11 .2017 